\title{
Lesão por pressão em pacientes acamados com idade avançada e os cuidados de enfermagem: Uma revisão integrativa da literatura
}

\author{
Pressure injury in patients with advanced age and nursing care: An integrative literature review \\ Lesión por presión en pacientes con edad avanzada y cuidados de enfermería: Una revisión \\ integrativa de la literatura
}

Recebido: 26/02/2021 | Revisado: 07/03/2021 | Aceito: 17/04/2021 | Publicado: 30/04/2021

\author{
Maria Inês Vieira de Oliveira Lima \\ ORCID: https://orcid.org/0000-0001-6696-904X \\ Centro Universitário do Estado do Pará, Brasil \\ E-mail: mariaignevieira@gmail.com \\ Jhully de Kassia Coutinho Pereira \\ ORCID: https://orcid.org/0000-0002-7833-3361 \\ Centro Universitário do Estado do Pará, Brasil \\ E-mail: jhullydekassia.jdk@gmail.com \\ Felipe Costa Soares \\ ORCID: https://orcid.org/0000-0003-4449-4324 \\ Centro Universitário do Estado do Pará, Brasil \\ E-mail: felipeesoaress@gmail.com \\ Mayra Gabriella do Nascimento Farias \\ ORCID: https://orcid.org/0000-0002-8324-8612 \\ Centro Universitário do Estado do Pará, Brasil \\ E-mail: fariasgabriella19@gmail \\ Eduarda Beatriz Reis Paz \\ ORCID: https://orcid.org/0000-0003-2910-6928 \\ Centro Universitário do Estado do Pará, Brasil \\ E-mail: reispaze@gmail.com \\ Alizandra Mendonça Reis \\ ORCID: https://orcid.org/0000-0001-8250-2714 \\ Centro Universitário do Estado do Pará, Brasil \\ E-mail: alizandra.99@hotmail.com \\ Elana Braga Costa \\ ORCID: https://orcid.org/0000-0002-1871-0727 \\ Centro Universitário do Estado do Pará, Brasil \\ E-mail: elana.braga@hotmail.com \\ Mônica Loureiro Maués Santos \\ ORCID: https://orcid.org/0000-0002-6478-396X \\ Centro Universitário do Estado do Pará, Brasil \\ E-mail: monicamlms@ outlook.com \\ Paula Sousa da Silva Rocha \\ ORCID: https://orcid.org/0000-0002-0453-1314 \\ Universidade do Estado do Pará, Brasil \\ E-mail:paulatuc@msn.com
}

\begin{abstract}
Resumo
Objetivo: Analisar na literatura estudos que abordem acerca da lesão por pressão em pacientes acamados com idade avançada. Método: Trata-se de um estudo do tipo revisão integrativa de literatura, de artigos publicados no período de 2016 a 2019, nas seguintes bases de dados: LILACS, SciELO e MEDLINE. Os termos para busca foram: Lesão por pressão; Cuidados de enfermagem; Idoso, publicados nos idiomas inglês e português. Foram identificados 165 artigos com temas pertinentes ao objetivo do estudo. Após a leitura na íntegra foram selecionados 20 artigos conforme a distribuição das publicações selecionadas nas bases de dados dos artigos escolhidos. Resultados: Tais publicações ressaltam sobre os cuidados de enfermagem em lesão por pressão em pacientes idosos, sobretudo na prevenção destas lesões, que são na maioria das vezes recorrentes em idosos acamados. Embora se reconheça as diversas causas da LPP, e a assistência multidisciplinar seja de total relevância para a o cuidado em saúde, é fato que a equipe de enfermagem é responsável pela assistência direta e contínua aos pacientes, o que lhe confere papel de destaque na prevenção desse problema. Conclusão: Evidenciou mediante a análise das produções selecionadas, que a lesão por pressão é mais comum
\end{abstract}


em pacientes acamados e idosos pela situação de vulnerabilidade. Isto ocorre mediante ao processo de envelhecimento, em que ocorrem várias alterações na pele em que suas estruturas de suporte associadas a outros sistemas corporais tornam a pele mais vulneráveis à pressão, cisalhamento e fricção. A enfermagem é essencial nas medidas preventivas, para assim reduzir e controlar os danos de lesão por pressão.

Palavras-chave: Lesão por pressão; Cuidados de enfermagem; Idoso.

\begin{abstract}
Objective: To analyze in the literature studies addressing pressure injuries in bedridden patients with advanced age. Method: This is an integrative literature review, of articles published from 2016 to 2019, in the following databases: LILACS, SciELO and MEDLINE. The search terms were: Pressure injury; Nursing care; Elderly, published in English and Portuguese. 165 articles were identified with themes relevant to the objective of the study. After reading in full, 20 articles were selected according to the distribution of the publications selected in the databases of the chosen articles. Results: Such publications emphasize nursing care for pressure injuries in elderly patients, especially in the prevention of these injuries, which are most often recurrent in bedridden elderly. Although the various causes of LPP are recognized, and multidisciplinary care is of utmost importance for health care, it is a fact that the nursing team is responsible for direct and continuous care for patients, which gives it a prominent role in preventing this. problem. Conclusion: It showed through the analysis of selected productions, that pressure injuries are more common in bedridden and elderly patients due to their vulnerability. This occurs through the aging process, in which there are several changes in the skin in which its support structures associated with other body systems make the skin more vulnerable to pressure, shear and friction. Nursing is essential in preventive measures, in order to reduce and control damage from pressure injuries.
\end{abstract}

Keywords: Pressure ulcer; Nursing care; Aged.

\title{
Resumen
}

Objetivo: Analizar en la literatura los estudios que abordan las lesiones por presión en pacientes encamados de edad avanzada. Método: Se trata de una revisión integradora de la literatura, de los artículos publicados de 2016 a 2019 , en las siguientes bases de datos: LILACS, SciELO y MEDLINE. Los términos de búsqueda fueron: lesión por presión; Cuidado de enfermera; Ancianos, publicado en inglés y portugués. Se identificaron 165 artículos con temas relevantes al objetivo del estudio. Después de la lectura íntegra, se seleccionaron 20 artículos según la distribución de las publicaciones seleccionadas en las bases de datos de los artículos elegidos. Resultados: Estas publicaciones enfatizan el cuidado de enfermería de las lesiones por presión en pacientes ancianos, especialmente en la prevención de estas lesiones, que son más recurrentes en ancianos postrados en cama. Si bien se reconocen las diversas causas de la LPP, y la atención multidisciplinar es de suma importancia para el cuidado de la salud, es un hecho que el equipo de enfermería es el responsable de la atención directa y continua al paciente, lo que le confiere un papel destacado en la prevención de este problema. Conclusión: Demostró a través del análisis de producciones seleccionadas, que las lesiones por presión son más comunes en pacientes encamados y ancianos debido a su vulnerabilidad. Esto ocurre a través del proceso de envejecimiento, en el que hay varios cambios en la piel en los que sus estructuras de soporte asociadas con otros sistemas corporales hacen que la piel sea más vulnerable a la presión, el cizallamiento y la fricción. La enfermería es fundamental en las medidas preventivas, con el fin de reducir y controlar los daños por lesiones por presión.

Palabras clave: Úlcera por presión; Atención de enfermaria; Anciano.

\section{Introdução}

A idade avançada produz modificações intensas no organismo humano, o que os tornam mais suscetíveis a doenças e lesões, podendo produzir sequelas e longos períodos de internação hospitalar. Os idosos se destacam, entre os indivíduos, por serem mais acometidos por doenças degenerativas, e por isso, necessitam ficar acamados, fato que, em muitos casos acarreta no desenvolvimento de lesões e ulceras de pressão (Lopes, 2017).

Conforme dados emitidos pela Sistema Único de Saúde (SUS), o cuidado de saúde destinado ao idoso é em relação às demais faixas etárias, o mais oneroso. Assim, 18,6\% do total de internações hospitalares foram registradas na faixa etária de 60 anos ou mais, para um segmento de idosos de apenas 8,5\% da população total do País; em comparação a 20,9\% de internações na faixa de zero a 14 anos, para uma população de 29,6\%; e 60,5\% de internações na faixa de 15 a 59 anos, que representam 61,8 da população total (Morosini et al., 2011).

Com esses parâmetros, é possível enxergar que os maiores índices de internação e ocupação de leitos hospitalares são de idosos. Assim, perceber-se que pela presença e tempo de permanência de idosos no intra-hospitalar, essa faixa etária desenvolve fatores de risco suscetíveis ao desenvolvimento de lesão por pressão (LPP), devido aos longos períodos de 
permanência em leitos, em muitas vezes, em uma única posição e sem mudança de decúbito. Atrelado a isso, o metabolismo de idosos são mais lentos, o tecido tegumentar é mais friável, a tonicidade muscular é mais enfraquecida e vulnerável, e por isso, desenvolve escaras (Araujo et al., 2019). As lesões por pressão (LPP) são caracterizadas por danos localizados na pele e/ou tecidos subjacentes, podendo ser sobre uma proeminência óssea, resultante de pressão isolada ou combinada com forças de cisalhamento e/ ou fricção, no qual somado a fatores intrínsecos os riscos são aumentados (Domansky \& Borges, 2014).

Existem dois fatores que contribuem para o risco de LPP: fatores Extrínsecos e intrínsecos. São fatores extrínsecos: umidade; calor; pressão, força de cisalhamento e fricção. Os intrínsecos: índice de massa corporal (IMC) $>30 \mathrm{Kg} / \mathrm{m} 2$ ou $<18,5$ $\mathrm{Kg} / \mathrm{m} 2$, anemia, deficiência nutricional proteica; extremos de idade, hipotensão arterial sistêmica, incontinência urinária/fecal, edema, hipertermia, tabagismo, desidratação; infecções sistêmicas ou locais; comorbidades crônicas (diabetes mellitus; imunossupressão; doenças renal, cardiovascular, neuromuscular, gastrointestinal e outras); uso de alguns tipos de medicamentos como: corticoides; sedativos; anestésicos; vasoativos (Hospital de Clínicas da Universidade Federal do Triângulo Mineiro, 2018).

Contudo, a enfermagem tem um papel imprescindível na prevenção dessa evolução clínica. Identificar e classificar o paciente com risco de LPP, classificando o risco individual e o perfil de risco por unidade; realizar a prescrição de cuidados e prevenção seguindo o Plano de Intervenções Institucional "Prevenção de Lesão de Pressão"; verificar o estado de conservação dos dispositivos de mobilização e redução de pressão; registrar o risco de LPP no quarto ou na beira do leito de cada cliente; capacitar a equipe de enfermeiros e técnicos quanto a medidas de adesão de prevenção e tratamento e ao preenchimento de formulários e registros. São medidas que, gradativamente, podem reduzir o risco de LPP dos pacientes (Fav reto et al., 2017).

Existem também protocolos terapêuticos para pacientes que evoluem, em seu caso clínico com a LPP. Registrar a presença de lesões no paciente, prescrever a terapia tópica, e o período da troca de curativos, conforme os protocolos do hospital ou de campo de prática; realizar o curativo e o desbridamento da ferida com instrumentos assépticos; avaliar e evoluir a lesão de cada troca de curativo dentro dos protocolos hospitalares. Além de participação da equipe multiprofissional que deve ser ativo nas capacitações de prevenção da LPP, e interagir do planejamento do âmbito hospitalar (Hospital de Clínicas da Universidade Federal do Triângulo Mineiro, 2018).

Dessa forma, o cuidado da enfermagem na prevenção, controle e tratamento na LPP, é imprescindível na evolução clínica e no prognostico do paciente. Além, da participação ativa do profissional enfermeiro nesse cuidado, a orientação e capacitação da equipe multiprofissional, em mostrar a importância da prevenção dessa evolução, as complicações advindas desse quadro e, principalmente, as medidas de intervenções hospitalares na não promoção deste quadro.

Partindo disso, desenvolveu-se uma Revisão Integrativa da Literatura (RIL) com objetivo de analisar na literatura estudos que abordem acerca da lesão por pressão em pacientes acamados com idade avançada visando contribuir o conhecimento diante de tal temática.

\section{Metodologia}

Trata-se de uma Revisão Integrativa da Literatura, de caráter exploratório-descritivo, com abordagem qualitativa, com evidencias a partir de materiais já publicados, constituído de artigos e periódicos disponibilizados nas bases de dados da internet. A revisão integrativa de literatura é um mecanismo característico, que tem como efeito reduzir os resultados adquiridos durante

o estudo sobre determinado tema ou problema de forma ordenada e extensa. É dada como integrativa por viabilizar os conhecimentos de forma mais abrangente de acordo com a pesquisa, gerando uma densa linha de conhecimento. Com isso, se torna livre ao pesquisador em efetuar uma revisão integrativa com distintos objetivos, conseguindo se voltar para descrição de conceitos, reavaliação de teorias ou pesquisa metodologica dos conhecimentos inclusos em um determinado tópico (Ercole, 2014). 
Para alcançar os objetivos proposto por esta pesquisa, foram utilizadas revisões literárias de artigo que apresentem como referências lesões por pressão em idosos e os cuidados de enfermagem referente a evolução de pacientes de idade avançada e acamados seguindo as seis fases do processo de construção do estudo: a identificação do tema e seus descritores indexados na base dos Descritores em Ciência em Saúde (DeCS); pesquisa na literatura e designar os critérios de inclusão e exclusão de estudos; definição dos objetos de estudos e informações extraídos das literaturas em análise; avaliação dos estudos incluídos; interpretação dos resultados, e por fim, a apresentação desta revisão (Sousa, 2018).

A coleta de dados foi realizada no segundo semestre de 2020, por meio das seguintes bases de dados: LILACS - Revisão latino-americana e do Caribe em ciência da saúde; SciElo - Scientific Eletronic Library Online; MEDLINE - Medical Literature Analysis and Retrieval System Online. Ademais foram utilizados para a busca dos artigos descritores cadastrados no DeCS (Descritores em Ciências da Saúde) e no MeSH (Medical Subject Heading), tais quais: Lesão por Pressão (Pressure Ulcer), Cuidados de Enfermagem (Nursing CARE), Idoso (Ages).

Quadro 1. Grupo e relação dos descritores para a pesquisa em banco de dados.

\begin{tabular}{|c|l|}
\hline GRUPOS & \multicolumn{1}{|c|}{ RELAÇÃO DOS DESCRITORES COM AS BASES DE DADOS } \\
\hline A & Revisão latino-americana e do Caribe em ciência da saúde \\
\hline B & Scientific Eletronic Library Online \\
\hline C & Medical Literature Analysis and Retrieval System Online \\
\hline
\end{tabular}

Fonte: Autores (2021).

A partir dos descritores, foram desenvolvidas três formas de pesquisa, sendo representado pelas letras: A, B e C. Cada grupo continha uma divisão que relacionava para uma pesquisa nas bases de dados (Quadro 1).

Como critérios de inclusão estavam: estudos completos com relevância científica de acordo com o tema nos idiomas português e inglês, disponibilizados com acesso aberto, obedecendo a um período de publicação de 2015 e 2019. Como critério de exclusão e eliminatório: produções incompletas com evidente fragilidade e sem conteúdo relacionado ao tema proposto. 
Figura 1. Fluxograma de busca e seleção de documentos.
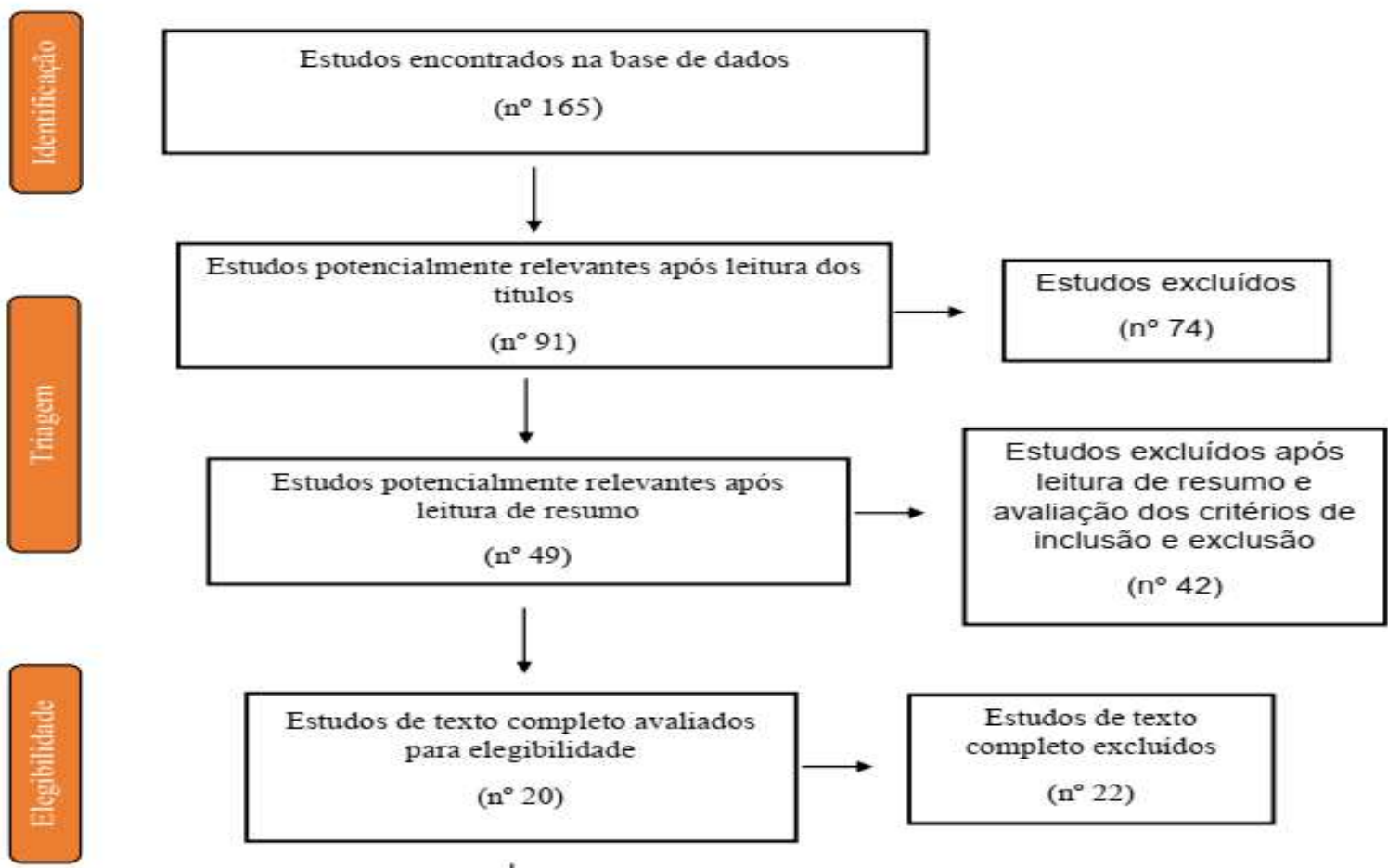

Estudos de texto completo avaliados para elegibilidade

$\left(n^{\circ} 20\right)$

Estudos de texto completo excluidos

$\left(n^{\circ} 22\right)$
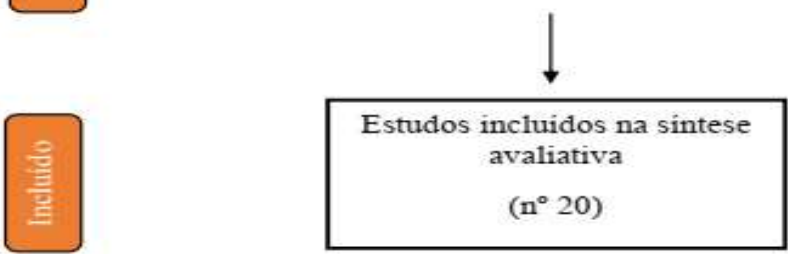

Fonte: Autores (2021).

Como demonstrado no fluxograma (Figura 1), o processo de seleção dos estudos para coleta de dados se obteve o total de 165 estudos nas referidas bases e banco de dados, no qual 91 estudos mostraram-se relevantes após leitura dos títulos e 74 foram excluídos. Após leitura 49 obtiveram relevância e 42 foram excluídos após avaliação nos critérios de inclusão e exclusão. $\mathrm{Na}$ análise final diante da leitura completa de texto foram incluídos 20 artigos para avaliação, sendo excluídos 22 restantes. Utilizou-se um instrumento para análise dos dados elaborados pelos pesquisadores deste trabalho e aplicado para cada artigo da amostra final dessa revisão, foi construído um quadro contendo as variáveis consideradas relevantes como: base de dados, título, autores, ano e o resultado principal.

\section{Resultados e Discussão}

Quadro 2. Artigos levantados nas bases de dados Lilacs, Scielo e Medline.

\begin{tabular}{|l|c|c|c|c|c|}
\hline $\begin{array}{l}\text { Base de } \\
\text { dados }\end{array}$ & Título & Autores & Ano & Idioma & Resultados \\
\hline Lilacs & $\begin{array}{c}\text { Fatores relacionados à } \\
\text { ocorrência de eventos } \\
\text { adversos em pacientes } \\
\text { idosos críticos }\end{array}$ & $\begin{array}{c}\text { Toffoletto MC, } \\
\text { Barbosa RL, Andolhe } \\
\text { R, Oliveira EM, } \\
\text { Janzantte Ducci A, } \\
\text { Padilha KG. }\end{array}$ & 2016 & Português & $\begin{array}{l}\text { Evidenciados os fatores diante dos eventos } \\
\text { adversos em pacientes idosos críticos, } \\
\text { estando presente LPP. }\end{array}$ \\
\hline
\end{tabular}




\begin{tabular}{|c|c|c|c|c|c|}
\hline Scielo & $\begin{array}{l}\text { Indicators of health and } \\
\text { safety among } \\
\text { institutionalized older } \\
\text { adults }\end{array}$ & $\begin{array}{c}\text { Cavalcante MLSN, } \\
\text { Borges CL, Moura } \\
\text { AMFTM, Carvalho } \\
\text { REFL }\end{array}$ & 2016 & Inglês & $\begin{array}{l}\text { A prevalência de úlcera por pressão variou } \\
\text { de } 8 \% \text { a } 23 \% \text {. Lesões por pressão são } \\
\text { indicadores importantes para detecção de } \\
\text { infecções em instituições de longa } \\
\text { permanência, mais comuns e que mais } \\
\text { acometem idosos. }\end{array}$ \\
\hline Scielo & $\begin{array}{l}\text { Ações de enfermagem } \\
\text { antes e após um } \\
\text { protocolo de prevenção } \\
\text { de lesões por pressão } \\
\text { em terapia intensiva }\end{array}$ & $\begin{array}{l}\text { Vasconcelos JMB, } \\
\text { Cariri MHL }\end{array}$ & 2017 & Português & $\begin{array}{l}\text { Após uso do protocolo, observou-se maior } \\
\text { frequência das ações: avaliação do risco para } \\
\text { lesões por pressão nos dias subsequentes à } \\
\text { admissão, observação de proeminência } \\
\text { ósseas e aplicação de hidratante, elevação do } \\
\text { paciente do leito na movimentação, proteção } \\
\text { de proeminência ósseas do joelho e elevação } \\
\text { do calcâneo. }\end{array}$ \\
\hline Medline & $\begin{array}{l}\text { Reducing Risks } \\
\text { Associated With } \\
\text { Negative-Pressure } \\
\text { Wound Therapy: } \\
\text { Strategies for Clinical } \\
\text { Practice. }\end{array}$ & Mattox EA & 2017 & Inglês & $\begin{array}{l}\text { Aborda os sistemas de terapia de feridas por } \\
\text { pressão negativa (NPWT), na qual } \\
\text { promovem a cicatrização de feridas por } \\
\text { atingir uma pressão negativa. }\end{array}$ \\
\hline Medline & $\begin{array}{l}\text { Adherence to evidence- } \\
\text { based pressure injury } \\
\text { prevention guidelines in } \\
\text { routine clinical practice: } \\
\text { a longitudinal study. }\end{array}$ & $\begin{array}{c}\text { Chaboyer W, } \\
\text { Bucknall T, Gillespie } \\
\text { B, Thalib L, McInnes, } \\
\text { E, Considine J, } \\
\text { Murray E, Duffy P, } \\
\text { Tuck M, Harbeck E. }\end{array}$ & 2017 & Inglês & $\begin{array}{l}\text { Dados sobre nove estratégias prevenção de } \\
\text { lesões por pressão baseadas em evidências, } \\
\text { uso de seis dispositivos de alívio de pressão } \\
\text { e uso de produtos para a pele, como cremes } \\
\text { de barreira. }\end{array}$ \\
\hline Lilacs & $\begin{array}{l}\text { Risco de lesão por } \\
\text { pressão em idosos com } \\
\text { comprometimento na } \\
\text { realização de atividades } \\
\text { diárias }\end{array}$ & $\begin{array}{l}\text { Vieira VAS, Santos } \\
\text { MDC, Almeida NA, } \\
\text { Souza CC, Bernardes } \\
\text { MFVG, Mata LRF. }\end{array}$ & 2018 & Português & $\begin{array}{l}\text { Verificou-se que } 64,3 \% \text { dos idosos possuíam } \\
\text { risco de desenvolver LP, e } 57,1 \% \text { eram } \\
\text { dependentes para realizar cinco ou mais } \\
\text { atividades de vida diária. Quanto maior a } \\
\text { independência para desenvolvimento de } \\
\text { atividades, menor o risco de desenvolver } \\
\text { UP. }\end{array}$ \\
\hline Lilacs & $\begin{array}{l}\text { Adesão da enfermagem } \\
\text { ao protocolo de lesão } \\
\text { por pressão em unidade } \\
\text { de terapia intensiva }\end{array}$ & $\begin{array}{l}\text { Sanches BO, Contrin } \\
\text { LM, Beccaria LM, } \\
\text { Frutuoso IS, Silveira } \\
\text { AM, Werneck AL. }\end{array}$ & 2018 & Português & $\begin{array}{l}\text { Dentre os } 945 \text { pacientes internados, apenas } \\
5,29 \% \text { apresentaram LPP durante a } \\
\text { internação, com predominância da faixa } \\
\text { etária de } 41 \text { a } 60 \text { anos e do sexo masculino. }\end{array}$ \\
\hline Lilacs & $\begin{array}{l}\text { The nurses' viewpoint } \\
\text { regarding the use of the } \\
\text { Braden scale with the } \\
\text { elderly patient }\end{array}$ & $\begin{array}{l}\text { Debon R, Fortes VLF, } \\
\text { Rós ACR, Scaratti M. }\end{array}$ & 2018 & Inglês & $\begin{array}{l}\text { Em grande maioria dos profissionais de } \\
\text { enfermagem conhecem e usam a escala de } \\
\text { Braden, porém encontram dificuldades para } \\
\text { que seja realmente efetivado os cuidados que } \\
\text { aparecem no escore de risco. }\end{array}$ \\
\hline Medline & $\begin{array}{l}\text { Prescription of pressure } \\
\text { injury preventative } \\
\text { interventions following } \\
\text { risk assessment: An } \\
\text { exploratory, descriptive } \\
\text { study. }\end{array}$ & $\begin{array}{c}\text { Lovegrove J, } \\
\text { Fulbrook P, Miles S. }\end{array}$ & 2018 & Inglês & $\begin{array}{l}\text { Abordagem estruturada para prescrição de } \\
\text { intervenção de acordo com o nível de risco, } \\
\text { pode ajudar a melhorar a prescrição de } \\
\text { intervenção preventiva dos enfermeiros e } \\
\text { garantir que todos os pacientes em risco } \\
\text { recebam intervenções preventivas } \\
\text { adequadas. }\end{array}$ \\
\hline Medline & $\begin{array}{l}\text { Prevention of heel } \\
\text { pressure ulcers among } \\
\text { adult patients in } \\
\text { orthopaedic wards: an }\end{array}$ & $\begin{array}{c}\text { Koh SY, Yeo HL, } \\
\text { Goh ML. }\end{array}$ & 2018 & Inglês & $\begin{array}{l}\text { A aplicação de protetores de espuma de } \\
\text { calcanhar é uma prevenção fundamental } \\
\text { medida de prevenção de lesões por pressão } \\
\text { no calcanhar no contexto local. }\end{array}$ \\
\hline
\end{tabular}




\begin{tabular}{|c|c|c|c|c|c|}
\hline & $\begin{array}{c}\text { evidence-based } \\
\text { implementation project. }\end{array}$ & & & & \\
\hline Scielo & $\begin{array}{l}\text { Lesão por Pressão em } \\
\text { Unidade de Terapia } \\
\text { Intensiva: estudo de } \\
\text { caso-controle }\end{array}$ & $\begin{array}{l}\text { Pachá HHP, Farias } \\
\text { JIL, Oliveira KA, } \\
\text { Bechara LM }\end{array}$ & 2018 & Português & $\begin{array}{l}\text { Entre os fatores de risco, destacaram-se, } \\
\text { idade maior ou igual } 60 \text { anos, internação por } \\
\text { doenças infecciosas, parasitárias e } \\
\text { neoplasias, períodos de internação maiores } \\
\text { que sete dias e estar internado em UTI que } \\
\text { não fosse UTI convênio. A maioria das } \\
\text { lesões foi notificada com grau de dano leve } \\
\text { e classificada em estágio II. }\end{array}$ \\
\hline Scielo & $\begin{array}{l}\text { Prevalence and factors } \\
\text { associated with chronic } \\
\text { wounds in older adults } \\
\text { in primary care }\end{array}$ & $\begin{array}{c}\text { Vieira CPB, Araújo } \\
\text { TME }\end{array}$ & 2018 & Inglês & $\begin{array}{l}\text { A prevalência de lesões entre idosos é alta } \\
\text { associado a maior ocorrência a } \\
\text { características socioeconômicas e clínicas. } \\
\text { Abordadas feridas crônicas no geral, } \\
\text { constatou-se que a prevalência da LPP é na } \\
\text { região sacral. }\end{array}$ \\
\hline Scielo & $\begin{array}{l}\text { Predição de risco de } \\
\text { lesão por pressão em } \\
\text { pacientes de unidade de } \\
\text { terapia intensiva: } \\
\text { revisão integrativa }\end{array}$ & $\begin{array}{l}\text { Zimmermann GS, } \\
\text { Cremasco MF, Zanei } \\
\text { SSV, Takahashi SM, } \\
\text { Cohrs CR, Whitaker } \\
\text { IY }\end{array}$ & 2018 & Português & $\begin{array}{l}\text { Entre as escalas genéricas predominou a } \\
\text { escala de Braden. Foram identificadas seis } \\
\text { escalas que apresentaram boa capacidade } \\
\text { preditiva para avaliar risco de lesão por } \\
\text { pressão em pacientes de unidade de terapia } \\
\text { intensiva. }\end{array}$ \\
\hline Scielo & $\begin{array}{l}\text { Prevenção de lesão por } \\
\text { pressão: ações } \\
\text { prescritas por } \\
\text { enfermeiros de centros } \\
\text { de terapia intensiva }\end{array}$ & $\begin{array}{c}\text { Mendonça PK, } \\
\text { Loureiro MDR, Frota } \\
\text { OP, Souza AS }\end{array}$ & 2018 & Português & $\begin{array}{l}\text { Foi encontrada associação estatística entre } \\
\text { as ações de mudança de decúbito, aplicação } \\
\text { de cobertura hidrocoloide em região sacral, } \\
\text { realização de higiene externa, troca de } \\
\text { fixação do cateter orotraqueal e/ou cateter } \\
\text { nasoenteral e inspeção da pele com a } \\
\text { ausência de lesões por pressão. A ocorrência } \\
\text { de lesões por pressão foi encontrada em } 49 \% \\
\text { dos clientes em ambas as instituições. }\end{array}$ \\
\hline Medline & $\begin{array}{l}\text { Clinical parameters of } \\
\text { wound healing in } \\
\text { patients with advanced } \\
\text { illness. }\end{array}$ & $\begin{array}{l}\text { Lai TT-K, Yip O-M, } \\
\text { Sham MMK. }\end{array}$ & 2019 & Inglês & $\begin{array}{l}\text { Lesões por pressão são propensas a não } \\
\text { cicatrizar em pacientes com idade avançada, } \\
\text { alto nível de creatinina, } \\
\text { estágio avançado da ferida, baixo PPS e } \\
\text { baixos escores de Norton. }\end{array}$ \\
\hline Medline & $\begin{array}{l}\text { Outcomes Associated } \\
\text { With Stage } 2 \text { Pressure } \\
\text { Injuries Among } \\
\text { Surgical Critical Care } \\
\text { Patients: A } \\
\text { Retrospective Cohort } \\
\text { Study }\end{array}$ & $\begin{array}{c}\text { Alderden J, Zhao YL, } \\
\text { Thomas D, Butcher R, } \\
\text { Gulliver B, Cummins } \\
\text { M. }\end{array}$ & 2019 & Inglês & $\begin{array}{l}\text { De } 6.376 \text { pacientes cirúrgicos em cuidados } \\
\text { intensivos, } 298 \text { desenvolveram lesão por } \\
\text { pressão adquirida no hospital estágio } 2 \text {. } \\
\text { Fatores inversamente relacionados à } \\
\text { presença de lesão por pressão adquirida } \\
\text { hospitalar curada foram idade avançada. }\end{array}$ \\
\hline Medline & $\begin{array}{l}\text { Preventive interventions } \\
\text { for pressure ulcers in } \\
\text { long-term older people } \\
\text { care facilities A } \\
\text { systematic review }\end{array}$ & $\begin{array}{l}\text { Mäki-Turja-Rostedt } \\
\text { S, Stolt M, Leino- } \\
\text { Kilpi H, Haavisto E. }\end{array}$ & 2019 & Inglês & $\begin{array}{l}\text { A incidência de úlcera por pressão nas } \\
\text { instalações de cuidados de idosos de longo } \\
\text { prazo diminuiu com o uso de decisão } \\
\text { computadorizada fazendo sistemas de apoio, } \\
\text { programas de prevenção, reposicionamento } \\
\text { ou avançado almofadas. A prevalência } \\
\text { diminuiu com programas de prevenção de } \\
\text { lesão, usando colchões e coberturas } \\
\text { avançadas ou adicionando suplementos de } \\
\text { proteína e energia a dieta. }\end{array}$ \\
\hline
\end{tabular}




\begin{tabular}{|c|c|c|c|c|c|}
\hline Scielo & $\begin{array}{l}\text { Permanent education } \\
\text { for good practices in the } \\
\text { prevention of pressure } \\
\text { injury: almost- } \\
\text { experiment }\end{array}$ & $\begin{array}{c}\text { Campo ALM, Engels } \\
\text { RH, Stacciarini TSG, } \\
\text { Cordeiro ALPC, Melo } \\
\text { AF, Rezende MP }\end{array}$ & 2019 & Inglês & $\begin{array}{l}\text { Estudo feito com enfermeiros acerca do } \\
\text { conhecimento dos mesmos em relação a } \\
\text { prevenção. }\end{array}$ \\
\hline Scielo & $\begin{array}{l}\text { Risk assessment for } \\
\text { perioperative pressure } \\
\text { injuries }\end{array}$ & $\begin{array}{l}\text { Peixoto CA, Ferreira } \\
\text { MBG, Felix MMS, } \\
\text { Pires OS, Barrichello } \\
\text { E, Barbosa MH }\end{array}$ & 2019 & Inglês & $\begin{array}{l}\text { A maioria dos pacientes apresentou alto } \\
\text { risco para lesão perioperatória por } \\
\text { posicionamento. Sexo feminino, idoso e } \\
\text { valores de índice de massa corporal } \\
\text { alterados foram estatisticamente } \\
\text { significativos. }\end{array}$ \\
\hline Scielo & $\begin{array}{l}\text { Avaliação do risco para } \\
\text { o desenvolvimento de } \\
\text { lesões perioperatórias } \\
\text { decorrentes do } \\
\text { posicionamento } \\
\text { cirúrgico }\end{array}$ & $\begin{array}{c}\text { Oliveira HMBS, } \\
\text { Santos AMJF, } \\
\text { Madeira MZA, } \\
\text { Andrade EMLR, Silva } \\
\text { GRF }\end{array}$ & 2019 & Português & $\begin{array}{l}\text { Foi identificado maior gravidade de lesão } \\
\text { correspondente ao uso de espuma e coxins } \\
\text { de algodão. Utilizou-se escala de Braden. }\end{array}$ \\
\hline
\end{tabular}

Fonte: Autores (2021).

Diante dos critérios de inclusão e exclusão previamente descritos nos artigos para a realização das buscas de acordo com os descritores, foram identificados 165 artigos com temas pertinentes ao objetivo do estudo. Após a leitura na íntegra foram selecionados 20 artigos conforme a distribuição das publicações selecionadas nas bases de dados dos artigos escolhidos. Deste modo, 4 foram encontrados na base de dados Lilacs, 7 na Medline e 9 na plataforma e Scielo.

Em relação ao ano de publicação, apenas 5 artigos foram publicados entre o ano de 2016 a 2017, porém nos anos seguintes (2018 a 2019) houve um grande índice de publicações referente ao tema em estudo, dando um total de 15 artigos publicados, o que demonstra um crescimento de publicações sobre LPP.

No que concerne aos métodos de estudos, 4 artigos são de método transversal, 3 em estudo longitudinal analítico, 2 em estudo documental, 2 em revisão integrativa da literatura, 2 em estudo exploratório, 2 estudos de coorte e os demais, em estudo baseado em evidências, estudos de caso, quase- experimental e pesquisa de campo, na qual são predominantemente da área da enfermagem.

Tais publicações ressaltam sobre os cuidados de enfermagem em lesão por pressão em pacientes idosos, sobretudo na prevenção destas lesões, que são na maioria das vezes recorrentes em idosos acamados. Embora se reconheça as diversas causas da LPP, e a assistência multidisciplinar seja de total relevância para a o cuidado em saúde, é fato que a equipe de enfermagem é responsável pela assistência direta e contínua aos pacientes, o que lhe confere papel de destaque na prevenção desse problema (Vasconcelos \& Cariri, 2017).

Um estudo realizado com idosos institucionalizados localizado no nordeste do Brasil, relatou que a LPP é um grande problema de saúde enfrentado nas instituições de longa permanência para idosos (Cavalcante, 2016). Isto é, há uma grande necessidade de estudos para investigar este acontecimento, pelo fato de que muitas vezes as lesões podem ser evitadas através da identificação precoce dos fatores de riscos existentes, assim como a implementação de medidas preventivas, tal estudo relata que a qualidade da assistência prestada pode ser medida de acordo com as ocorrências de lesão, ou seja, quanto menor o número de casos, melhor é a assistência prestada aqueles pacientes (Vieira \& Araújo, 2018). Portanto, de maneira geral, para uma boa assistência de enfermagem, é preciso utilizar cada vez mais medidas preventivas (Lai; Yip \& Sham, 2019).

Em relação a classificação de risco, após a análise foi possível observar que $70 \%$ dos estudos estão voltados para este tema, dentre eles, utilizaram a escala de Braden como método de avaliação para o risco de lesões por pressão, esta escala é um instrumento muito útil, de fácil manuseio, não tendo custo para a instituição e usado como um indicador de saúde e na segurança 
do paciente, portanto, auxilia o enfermeiro na realização da avaliação dos riscos do paciente em relação a formação de lesão (Debon et al., 2018).

Assim, o seu bom uso contribui para que sejam realizados cuidados adequados de acordo com a realidade de cada paciente, para que assim LPP possa ser evitada. Porém, de acordo com um estudo realizado com enfermeiros de um determinado hospital no Rio Grande do Sul, observou-se que alguns enfermeiros ainda não utilizam a escala de Braden, ou utilizam de forma incorreta. Em contrapartida, foi possível observar que a LPP acaba sendo negligenciada por muitos enfermeiros, ou seja, algo que eles já esperam que aconteça (Sanches et al., 2018). Além disso, muitos realizam o preenchimento da escala de Braden sem avaliar presencialmente o paciente.

Durante a análise, observou-se que em dois artigos sobre LPP decorrente de posicionamento cirúrgico, o fator de risco que mais influência na ocorrência de lesão é o tempo de duração do procedimento anestésico cirúrgico, visto que, a sedação e anestesia impossibilitam o paciente de sentir dor ou mudar de posição durante o procedimento cirúrgico, logo bloqueiam a sensibilidade do corpo à dor e à pressão, causando vasodilatação e consequentemente redução da pressão arterial e perfusão tecidual. Assim, a pressão em áreas do corpo, causam a ruptura do tecido e consequentemente a lesão por pressão (Oliveira et al., 2019; Peixoto et al., 2019; Alderden et al., 2019).

Quanto aos pacientes acamados em terapia intensiva, 5 estudos apresentaram que pacientes nessa situação apresentam elevado risco para o desenvolvimento de LPP, isso correspondente à utilização de equipamento respiratório, cateteres urinários, dispositivos de compressão sequencial, múltiplos cateteres intravenosos e a infusão de drogas vasoativas, esses equipamentos levam à diminuição da percepção sensorial causada por sedativos, analgésicos e relaxantes musculares, fator predisponente a geração de lesão (Pachá et al., 2018)

A utilização de drogas vasoativas e a ventilação mecânica invasiva não apontaram associação significativa entre clientes com e sem LP, por outro lado, o estudo defende que pesquisas mostraram que a internação e o uso de drogas vasoativas podem contribuir para a gênese das LP. Todavia, são necessários estudos mais aprofundados sobre essa temática, ademais, a avaliação clínica, juntamente com a utilização de tecnologias preventivas de LPP, devem ser implementadas em todos pacientes que obtiverem riscos (Mendonça et al., 2018).

Uma pesquisa realizada em um hospital de ensino de porte especial do interior de São Paulo, em seis UTIs, declarou que ocorrência de LPP prolonga a hospitalização e consequentemente os custos do tratamento, além de aumentar ainda mais as chances de o paciente desenvolver complicações e infecções, fato que dificulta a recuperação e representa um acréscimo no sofrimento emocional e físico dos pacientes (Zimmermann et al., 2018).

No que se refere a localização das LPs, as áreas mais frequentes nos estudos são a região sacral, cóccix, crista ilíaca, glúteo, calcanhar, região occipital, cotovelos e panturrilhas. Tais regiões foram apontados como os locais onde mais ocorrem lesão por pressão em pacientes acamados (Mendonça et al., 2018). Constatou-se também, que essas regiões são mais afetadas por consequência do frequente posicionamento do paciente em decúbito dorsal.

O que naturalmente é a posição do corpo quando em repouso, porém deixa regiões com proeminências ósseas em contato com a superfície, o que, se não manejado corretamente pode levar a ocorrência da LPP. Deste modo, para prevenir a ocorrência de lesão é importante reduzir o tempo que o paciente permanece em determinado posicionamento, realizando mudanças de posição em horários programados (Toffoletto et al., 2016). Outro fator também apontou a importância de ações que reduzam o tempo de permanência do paciente na instituição (Chaboyer et al., 2017).

Dentre os cuidados de enfermagem mais utilizados, 5 artigos afirmaram a utilização de dispositivos de alívio de pressão, como travesseiro para elevar o calcanhar, colchão de ar, almofadas, protetores de espuma, cunhas, botas de cotovelo e outros dispositivos para prevenção de LPP. O uso de travesseiros e cunhas reduz a pressão sobre proeminências ósseas, em especial 
quando utilizados entre superfícies da pele, como joelhos, onde pode haver o atrito, e sob panturrilhas, tais dispositivos reduzem a pressão da superfície com o calcanhar (Koh; Yeo \& Goh, 2018)

Para Vieira et al (2018), essas medidas são de total responsabilidade da equipe de enfermagem e configuram-se como essenciais para garantir a segurança do paciente e prevenir LPP. Outros cuidados de enfermagem muito utilizados foram a observação de pele e proeminência óssea, elevação de calcanhar e da cabeceira a 30 graus e mudança de decúbito, na qual foram citados pela maioria dos artigos em estudo (Mäki-Turja-Rostedt et al., 2019).

A mudança de decúbito a cada duas horas é uma das principais medidas para prevenção de LPP, pois isso evita a redução do fluxo sanguíneo do tecido, que por um período prolongado de tempo pode ocasionar lesão. Este estudo relatou que na instituição do estudo, existe nas UTIs o relógio de mudança de decúbito, que é um impresso plastificado, na qual indicam em qual posição o paciente precisa estar naquela determinada hora, desta forma, os profissionais podem se atentar para o horário em que devem realizar o procedimento de prevenção, um método que é eficaz naquela instituição e que poderia ser implementado em outros locais onde tenham pacientes acamados (Sanches et al., 2018).

Além do mais, cuidados como a aplicação de hidratante corporal para manter a pele hidratada, realizar higiene externa, como a troca de fraudas com frequência e realização de higiene externa, bem como orientar o paciente quanto a importância da mesma, foram os cuidados de enfermagem vistos com menos frequência, mas que são de grande importância ao cuidado do paciente idoso (Vasconcelos \& Cariri 2017).

Outra questão muito importante a ser realizada é a capacitação profissional e educação contínua dos profissionais, segundo o autor Pachá et al (2018), a capacitação profissional é também uma forma de cuidado, tanto na prevenção quando no tratamento. Desse modo 2 artigos abordaram acerca da importância da prevenção de lesão por pressão através da educação continuada (Campo et al., 2019; Mattox, 2017).

Acerca da educação do paciente sobre o risco de desenvolver LPP, após o enfermeiro concluir a avaliação de risco e identifica-lo, é importante orientar o paciente e/ou acompanhante, sobre o risco que o paciente está exposto, como por exemplo, distribuindo apostilas com informações pertinentes a prevenção da LPP, e a partir disso, discutir os cuidados que serão realizados, afim de evitar que futuramente o paciente obtenha algum tipo de lesão (Lovegrove; Fulbrook \& Miles, 2018).

\section{Conclusão}

Com este estudo, conclui-se, que a lesão por pressão é mais comum em pacientes acamados e idosos pela situação de vulnerabilidade. Isto acontece porque o processo de envelhecimento do idoso facilita a ocorrência de alterações na pele, nas quais suas estruturas de suporte associadas a outros sistemas corporais tornam a pele mais vulneráveis à pressão, cisalhamento e fricção. Além disso, pessoas com idades avançadas que possuem um longo período de internação, tornam-se mais suscetíveis ao desenvolvimento de LPP.

Os enfermeiros são os profissionais mais ativos no cuidado de LPP, pois utilizam-se de escalas para avaliar o grau de risco da lesão e reconhecimento dos indivíduos capazes de desenvolve-las, além de atuar de maneira intensa no tratamento. No entanto, é notório que os instrumentos utilizados no cuidado não são normatizados, assim, trazendo um possível prejuízo a assistência prestada (Vieira et al., 2018). Em função disso, ressalta-se a importância da padronização da atenção ofertada por esses profissionais no tratamento de pessoas com LPP, com o objetivo de diminuir a ocorrência desses agravos e proporcionar maior qualidade de vida. 


\section{Referências}

Alderden, J. et al (2019). Outcomes Associated With Stage 2 Pressure Injuries Among Surgical Critical Care Patients: A Retrospective Cohort Study. Critical care nurse, 39(4), 13-19.

Araújo, S. S. et al (2019). Prevenção de Lesões Por Pressão em Idosos: o cuidar do profissional de Enfermagem. Rev. FEVISP, $291-305$.

Cavalcante, M. L. S. N. et al (2016). Indicators of health and safety among institutionalized older adults. Rev. esc. enferm. USP, 50(4), 602-609.

Campo, A. L. M. et al (2019). Permanent education for good practices in the prevention of pressure injury: almost-experiment. Rev Bras Enferm., 72(6):172531.

Chaboyer, W. et al (2017). Adherence to evidence-based pressure injury prevention guidelines in routine clinical practice: a longitudinal study. Rev. Int Wound J., 14(6), 1290-1298.

Debon, R. et al (2018). The nurses' viewpoint regarding the use of the Braden scale with the elderly patient. Rev. Onl. De Pesquisa Cuidado é Fund, 10(3), 8117823.

Domansky, R. C. \& Borges, E. L. (2014). Manual para prevenções de lesão de pele. Rubio,

Ercole, F. F.; Melo L. S.; Alcoforado C. L. G. C (2014). Revisão integrativa versus revisão sistemática. Revista Mineira de Enfermagem, $18,9-11$.

Favreto, F. J. L. et al (2017). O papel do Enfermeiro na Prevenção, Avaliação e Tratamento das Lesões por Pressão. Rev. Gestão \& Saúde, $17(2), 37-47$.

Hospital de Clínicas da Universidade Federal do Triângulo Mineiro (2018). Ministério da Educação. Protocolo assistencial multiprofissional: Prevenção e tratamento de lesão por pressão. Uberaba-MG: Serviço de Educação em Enfermagem.

Koh, S. Y., Yeo, H. L. \& Goh, M. L (2018). Prevention of heel pressure ulcers among adult patients in orthopaedic wards: an evidence-based implementation project. International journal of orthopaedic and trauma nursing, 31, 40-47.

Lai, T. T., Yip, O. \& Sham, M. M. K. (2019). Clinical parameters of wound healing in patients with advanced illness. Annals of palliative medicine, 8(1), 5-14.

Lopes, L. S. \& Spolidoro, F. V. (2017). O papel do enfermeiro na prevenção e cuidado da úlcera por pressão no paciente idoso acamado. Rev. Enfermagem em Evidência, 1(1), 19-32.

Lovegrove, J.; Fulbrook, P. \& Miles, S. (2018). Prescription of pressure injury preventative interventions following risk assessment: An exploratory, descriptive study. International wound journal, 15(6), 985-992.

Mäki-Turja-Rostedt, S. et al (2019). Preventive interventions for pressure ulcers in long-term older people care facilities: A systematic review. Journal of clinical nursing, 28(13-14), 2420-2442.

Mattox, E. A. (2017). Reducing risks associated with negative-pressure wound therapy: strategies for clinical practice. Critical care nurse, 37(5), 67-77.

Mendonça, P. K. et al (2018). Prevenção de lesão por pressão: ações prescritas por enfermeiros de centros de terapia intensiva. Rev. Texto Contexto Enfermagem, 27(4).

Morosini, S. et al (2011). Custo e tempo de permanência hospitalar de idosos residentes em Recife-PE. Rev. Geriatria \& Gerontologia, 5(2), 91-98.

Oliveira, H. M. B. S. et al (2019). Avaliação do risco para o desenvolvimento de lesões perioperatórias decorrentes do posicionamento cirúrgico. Rev. Gaúcha Enfermo, 40.

Peixoto, C. A. et al (2019). Classificação de risco de desenvolvimento de lesões decorrentes do posicionamento cirúrgico. Rev. Latino-Am. Enfermagem, 27.

Pachá, H. H. P. et al (2018). Pressure Ulcer in Intensive Care Units: a case-control study. Rev. Brasileira de Enfermagem, 71(6), 3027-3034.

Sanches, B. O. et al (2018). Adesão da enfermagem ao protocolo de lesão por pressão em unidade de terapia intensiva. Arquivos de Ciências da Saúde, 25(3), $27-31$.

Sousa, L. M. M. et al (2018). Revisões da literatura científica: tipos, métodos e aplicações em enfermagem. Revista Portuguesa de Enfermagem de Reabilitação, $1(1), 45-54$

Toffoletto, F. C. et al (2016). Fatores relacionados à ocorrência de eventos adversos em pacientes idosos críticos. Rev. Brasileira de Enfermagem, 69(6), 1103445 .

Vasconcelos, J. M. B. \& Cariri, M. H. L. (2017). Ações de enfermagem antes e após um protocolo de prevenção de lesões por pressão em terapia intensiva. Rev. Esc. Anna Nery, Rio de Janeiro, 21(1).

Vieira, V. A. S. et al (2018). Risco de lesão por pressão em idosos com comprometimento na realização de atividades diárias. Rev. Enfermagem do Centro-Oeste Mineiro, 8 .

Vieira, C. P. B. \& Araújo, T. M. V. (2018). Prevalence and factors associated with chronic wounds in older adults in primary care Prevalencia y factores asociados con heridas crónicas en ancianos en la atención básica. Rev. Esc. Enferm USP, 52.

Zimmermann, G. S. et al (2018). Predição de risco de Lesão por Pressão em pacientes de Unidade de Terapia Intensiva: Revisão Integrativa. Rev. Texto Contexto Enferm, 27(3). 